\title{
L'institution des sujets: essai de dépassement du dualisme et de l'influence du néolibéralisme dans les sciences humaines
}

Laurence Gavarini *

\begin{abstract}
RESUMO
O trabalho traz um debate sobre alguns dualismos presentes nas ciências humanas, tais como aqueles entre os conceitos de indivíduo e sociedade, sujeito e instituição, ou entre os campos referências da sociologia e da psicanálise. Para tanto, analisa a noção de instituição como dinâmica e a concepção de sujeito na psicanálise, situando as discussões no terreno do neoliberalismo contemporâneo, que promove um indivíduo todo poderoso, dono de seu destino, criado na cultura do individualismo. Partindo da crítica das instituições violentas e das forças de opressão, chega até a crise contemporânea das subjetividades, propondo a desconstrução das polarizações.
\end{abstract}

Palavras-chave: Ciências humanas. Dualismos. Neoliberalismo.

\section{The institution of sujects: an attempt to overcome dualism and the influence of neoliberalism in human sciences}

\begin{abstract}
The article proposes a debate on the dualisms found in human sciences, in concepts as individual and society, subject and institution, or in the reference fields of sociology and psychoanalysis. It analyses the notion of institution as being dynamic and the concept of subject in psychoanalysis, locating the discussion within the contemporary neo-liberalism, which promotes the almighty individual, master of his own destiny, created in the culture of individualism. Starting with the critique of the violent institutions and the forces of oppression, it ends up with the discussion of the contemporary crisis of subjectivities, and proposes de deconstruction of polarizations.
\end{abstract}

Keywords: Human sciences. Dualism. Neo-liberalism.

* Sociologue, maître de conférences en Sciences de l'Education (Université de Paris 8), est l'auteur de La passion de l'enfant: filiation, procréation et éducation à l'aube du XXIème siècle. Paris: Denoël, 2001; (avec Françoise Petitot); La fabrique de l'enfant maltraité: un nouveau regard sur l'enfant et la famille. Ramonville Sainte-Agne: ERES, 1998.E-mail: yabakar@univ-paris8.fr 
Mon propos ne peut s'envisager que dans un entre-deux; entre deux champs référentiels, la sociologie, la psychanalyse; entre deux concepts, l'institution, le sujet; entre le plan des subjectivités et celui de la scène sociale; aux frontières de l'individu et de la société, du champ social et de l'inconscient. En réfutant ces grands dualismes, construits et maintenus fréquemment comme tels sur un mode le plus souvent exclusif dans les sciences humaines, ${ }^{1}$ et en travaillant à ce niveau d'entrelacs nous nous trouvons dans une zone où se manifestent de sérieuses turbulences théorico-pratiques!

Il n'est guère concevable de procéder par compilation des savoirs de ces deux disciplines à la fois proches et lointaines car ceux de l'une dérangent, voire subvertissent, ceux de l'autre et réciproquement. Par exemple, cela représente une sérieuse difficulté d'articuler sujet social et sujet de la psychanalyse, autrement dit, de penser ensemble l'acteur social, sujet de l'action, sujet de droits, sujet démocratique et le sujet de l'inconscient, un sujet qui n'est pas non plus le moi psychologique, mais un sujet divisé. On ne peut que se dire en effet qu'il y a sujet et sujet. Et nous prenons toujours le risque d'installer une certaine 'confusion des langues', à défaut de pouvoir appréhender leurs points d'achoppement ou, le cas échéant, leur dialectique.

Autrement dit, le contenu même de l'idée d'institution des sujets oblige à travailler au cœur d'une tension où il s'agirait de penser une sociologie des subjectivités ou une psychanalyse du lien social. Essayons de déployer cette tension, de reprendre ses divers pôles tels qu'ils se sont manifestés et élaborés dans le temps, tels du moins que nous pouvons aujourd'hui les reconstruire en retraçant imparfaitement, certes, mais aidés par la distance de l'après coup, les théories tant de l'institution que du sujet qui marquèrent le parcours intellectuel d'une génération de sociologues formés aux lendemains de $1968 .^{2}$

\section{LA CRITIQUE DES INSTITUTIONS COMME FORMES OPPRESSIVES}

Les années 1970 ont été significativement marquées dans leur approche des institutions et des faits sociaux par une sociologie critique des formes instituées et bureaucratiques: celles-ci, sont incarnées par les institutions qui opacifient aux yeux des individus le sens de leur action et de leur inscription ainsi que leurs relations, et rendent le pouvoir captif d'une minorité. Cette origine a été déterminante dans la construction de représentations opposant les institutions et les sujets, sur le mode d'une césure ou d'une discontinuité radicale.

La critique des dimensions contraignantes, répressives et coercitives domine de nombreux écrits théoriques de la sociologie à la psychosociologie en passant par la philosophie. Dans la période, tous prennent une coloration très politique.

Pour résumer schématiquement: l'institution était décrite comme "institution totale" par Goffman (1968) dont les travaux nous ont amenés à considérer les institutions asilaires comme étant une sorte de parangon ou d'archétype; elle se matérialisait dans une organisation panoptique, des techniques de contrôle et de répression, tant pour Michel Foucault (1975) penseur de la prison que pour Robert 
Castel (1976), théoricien du médico-social et de la psychiatrie qui en ont fait ressortir la dimension disciplinaire. Ces "machines" à fabriquer de la subjectivation et de la territorialisation, pour Guattari (1995), devenaient, avec l'école, des "casernes", selon Fernand Oury et Jacques Pain (1972), un système de sélection, de reproduction sociale et de violence symbolique pour Bourdieu. C'est sous le concept d' "institutions de la violence", que Franco Basaglia (1976), théoricien italien de l'anti-psychiatrie, a tenté par ailleurs d'épingler un trait commun à l'ensemble des institutions sociales.

Bref, cette concentration de lectures nous a fortement imprégnés en tant que génération. Bien sûr la violence institutionnelle réelle et symbolique caractérisait plus certaines institutions d'éducation, de soin ou d'enfermement. Mais c'est plus globalement la forme sociale institution elle-même sur laquelle portait une critique tour à tour idéologique et théorique. Il allait de soi, alors, que l'oppression ne supposait pas un fonctionnement disciplinaire caractérisé ou manifeste, elle pouvait s'exercer tout aussi bien selon des modes plus discrets, sur les individus dans l'ensemble des institutions de la société..

Les mots utilisés venaient à l'appui de la démonstration du caractère mécanique, si ce n'est quasiment physique, des institutions: elles sont des équipements du pouvoir, des appareils, des machines, des dispositifs constituant un système normatif et une emprise sur les individus, voire un pur jeu de forces et de formes sociales antagonistes, etc. Elles ont pour fonction ou pour effets la 'normalisation' des comportements et des agires, synonymes, dans les esprits 'révolutionnaires' de l'époque de mise au pas et de standardisation. Du même coup, elles paraissent ne pas pouvoir échapper à une logique d'aliénation de leurs ressortissants, tout comme Marx avait pensé l'aliénation par le travail au milieu du XIXème siècle.

Les façons de penser tant la société que le lien social, ou même les relations intersubjectives, découlaient de cette série de représentations: les institutions étaient des ensembles uniformisants si ce n'est uniformes. On les critiquait en raison de leur dimension structurale, c'est-à-dire de leurs invariants formels, de leur pérennisation, de leur conservatisme, de leurs interconnexions venant à l'appui d'un système forcément opposé aux individus, à l'initiative et aux libertés.

$\mathrm{Au}$ fond, on les critiquait pour ce qui avait amené notamment Durkheim (1949), en d'autres temps, à leur accorder, dans Les règles de la méthode sociologique, une réelle fonction sociale, c'est-à-dire une fonction de socialisation voire de prévention de l'anomie.

\section{L'INSTITUTION COMME DYNAMIQUE}

La conception dialectique et politique de l'institution proposée par Lourau $(1969,1970)$ et par Castoriadis (1975) se détachait toutefois singulièrement de cet ensemble de travaux sociologiques critiques. Leurs enseignements au long des années 1970 prédisposaient à une lecture d'emblée moins massifiante ou pétrifiante de la question de l'institution, quand bien même attiraient-ils l'attention sur ses effets répressifs. 
Côté théorique, ces auteurs, tout en partageant l'essentiel des présupposés de leurs contemporains, ont contribué, chacun à sa façon, à faire surgir la dimension conflictuelle cachée de toute institution. En fait leur théorisation était directement en prise sur les mouvements sociaux de cette époque concernant les prisons, les asiles, l'école ou encore la santé, et ils ont su interpréter ce que ces mouvements avaient révélé au grand jour: c'est-à-dire les forces instituantes, les collectifs et les groupes à l'œuvre dans les institutions faisant que celles-ci ne sauraient être réduites à leur forme administrative instituée (organisation, règles, fonction sociale, etc.). Leur conception de l'institution constituait pour nous un regard novateur sur la société. Elle établissait que la société, le social ne pouvaient se résumer à ce que l'on peut en saisir à travers les concepts de stratification et de classes sociales, ou par la théorie de l'acteur et du système, ou encore par les notions d'interactions et de rôles sociaux. La société et le social se distribuent dans les institutions et s'y ordonnent. Et en dépit de cohésions groupales internes fortes (qu'un concept comme celui de classes institutionnelles élaboré par Gérard Mendel (1975) permet par ailleurs d'identifier), des tensions les travaillent de l'intérieur. Autrement dit, elles sont traversées par des intérêts singuliers qui agissent contre leurs objectifs universels (LOURAU, 1969, 1970).

Côté politique et clinique (l'un et l'autre étant articulés), le changement escompté de la pratique collective de l'analyse institutionnelle concernait prioritairement les rapports d'oppression et de domination au sein de l'institution; les contraintes et les normes étant considérés comme des vecteurs de l'institué. Conséquemment, il n'était pas d'impositions qui ne méritaient d'être discutées, de lois qui valaient dans l'absolu, de règles administratives et organisationnelles incontestables, de limites qui ne soient arbitraires, de pouvoirs qui ne soient assimilés à une volonté de contrôle ou d'emprise. Du moins est-ce sous cette forme radicale que la critique de l'institué s'est développée à travers l'intervention socianalytique.

L'analyse institutionnelle en tant que sociologie d'intervention n'avait guère de considération in situ pour la question de l'individu, et a fortiori pour celle du sujet. ${ }^{3} \mathrm{Du}$ moins, l'individu n'était-il pas autrement appréhendé dans l'analyse qu'en ce qu'il pouvait éclairer - en tant qu' analyseur - une situation institutionnelle. Il apparaissait pour s'être singularisé par son action et par son expression inattendues dans le groupe, par les manifestations de sa déviance ou par son exclusion. Au total, l'attention était surtout portée sur les groupes et les collectifs composant les institutions et agissant sur leurs mécanismes, les transformant de l'intérieur, ${ }^{4}$ les sujets n'en étant que des éléments moteurs ou résistants. On reconnaissait que les personnes étaient mûes par des facteurs internes (leurs implications, dont Lourau théorisait la portée), mais aussi par des facteurs externes à la situation (la transversalité inventée par Guattari) lesquels agissaient sur lui et en lui à la façon de quasi déterminismes ou de déterminants socio-subjectifs. La subjectivité de cet individu impliqué se présentait comme une série de plis et de replis, agissant dans une certaine ombre son rapport aux institutions et aux actes, en même temps que l'institution était représentée comme agissant les siens, les faisant en quelque sorte se plier. Cette représentation n'était pas très éloignée de celle de Deleuze lorsqu'il 
envisageait le pli subjectif du pouvoir en chaque individu. Reste que les individus ne sauraient être réduits à leur assujettissement par la "société de contrôle" telle que la décrit Deleuze (1990), lorsqu'ils sont considérés par ailleurs comme étant porteurs de l'instituant dans les institutions dont ils sont membres.

La lecture se complexifiait en intégrant cette représentation selon laquelle les institutions ne font pas que répondre à des besoins ou à des nécessités pratiques immédiates, mais remplissent aussi des fonctions symboliques et imaginaires, telles qu'identifiées par Castoriadis (1975). Ce dernier insistait dans ses écrits sur la tension existant dans toutes les institutions de la société entre un imaginaire effectif, qui contribue à la reproduction, et un imaginaire radical, propice à la création de nouvelles significations. Cette dimension créative était centrale dans sa théorie, justifiant l'usage qu'il faisait du devenir à la fois au plan des sujets et à celui de la société. ${ }^{5}$

En élevant l'institution au niveau des significations auxquelles tout sujet doit se confronter, Castoriadis en fait donc une instance d'abord symbolicoimaginaire. Dans le même mouvement, il extra territorialise, délocalise en quelques sortes ses fonctions, celles-ci sont moins directement tangibles dans les pratiques ou les actes, qu'elles ne peuvent être appréhendées au travers du sens et de la subjectivation, c'est-à-dire de la manière dont les significations sont intériorisées et/ou rejetées par les individus, dont elles font ou non identité commune. C'est ce que l'analyse institutionnelle, en dépit de son projet explicite de comprendre les formes d'actualisation de l'institué et de l'instituant, n'a jamais vraiment atteint. La raison en est double. D'une part, elle est restée très attachée à la "base matérielle", aux lieux, aux pratiques concrètes et aux relations de pouvoir et peu à même d'appréhender ces autres dimensions plus "immatérielles" que sont l'imaginaire et le symbolique. D'autre part, son modèle de compréhension a toujours été centré sur la négativité institutionnelle (théorie des analyseurs) telle qu'elle s'exprime dans la dissension, le dysfonctionnement, le conflit; l'unité positive étant, sinon désaffectée, peu utilisée comme levier de l'analyse.

C'est à notre sens une contradiction majeure que d'avoir mis le "travail du négatif" au principe même de son modèle opératoire (en se focalisant toujours sur la mise en évidence de ce qui désassemble les groupes, ce qui désunit les pratiques, ce qui divise les individus, ce qui découd les rationalités), tout en se revendiquant (est-ce un idéal?) d'une clinique de la résorption du conflit institutionnel. En faisant ainsi miroiter une institution unifiée, rassemblée, voire réconciliée, les praticiens de l'analyse institutionnelle sembleraient ne pas avoir échappé à un imaginaire pastoral concernant leur mission!

\section{LE DENI DES FORCES ANOMIQUES}

En repensant aujourd'hui à mon éloignement, si ce n'est ma rupture, avec le paradigme critique de la fonction oppressive des institutions, il s'est joué sur les deux plans théorique et idéologique.

Au plan idéologique ou politique, il me semble que la solidarité permanente et aujourd'hui encore active entre le concept d'institution et celui d'oppression, 
qui justifie la subversion de tout institué, a toujours été un point d'aveuglement. Car, en effet, si l'on se range sous un paradigme plus anthropologique, il est rapidement clair que la fonction subjectivante et socialisatrice que doit remplir l'institution en tant qu'instance tierce et extérieure au sujet lui faisant limite, ne saurait, contrairement à ce que laisse entendre une critique obtuse des institutions, relever d'une logique d'arrangements interindividuels ou de règles consensuelles et contractuellement consenties. La mise en accusation a priori de l'institué, dont les individus seraient forcément "victimes" face à un pouvoir toujours abusif et maltraitant, au lieu de les protéger, confine à la perte de significations imaginaires communes. C'est ce que donne à voir le déchaînement des haines et de la violence, lorsque ce qui tient lieu, dans une institution, de régulation entre les sujets n'est plus que la loi des uns contre les autres, la loi du "plus fort" devenant alors la règle... Il n'y a manifestement plus guère de courant de pensée où l'on ignorerait encore aujourd'hui que le maniement des conflits, érigé comme principe de régulation, met aux commandes perversions et démagogies, si ce n'est folie. $\mathrm{Au}$ demeurant, l'obstination anti-institutionnelle finit par rejoindre, dans le temps, un phénomène social de plus grande ampleur, caractéristique du néolibéralisme régnant, à savoir un amollissement généralisé de la forme institution.

Au plan théorique, des problèmes majeurs se sont posés d'emblée et demeurent irrésolus à ce jour: tout d'abord la fonction heuristique centrale prêtée aux analyseurs qui repose sur l'hypothèse que les dysfonctionnements et les transgressions révèlent forcément les contradictions et le sens caché de l'institution, et non pas son non-sens ou le dysfonctionnement des siens; et ensuite le parti pris, ingénu, en faveur de l'instituant en toutes circonstances qui débouche sur le fait de donner une valence positive à toutes les forces d'opposition ou de rupture se manifestant dans l'institution, même celles qui, précisément, entraînent du côté de l'anomie, du pur rapport de forces ou du chaotique. Cet apriorisme construit l'instituant - innovateur par définition - comme menacé de répression par un institué conservateur, bureaucratique ou réactionnaire (anathème redevenu très à la mode ces derniers temps). Cette lecture n'a rien, contrairement à ce qui est dit, de dialectique mais renvoie une nouvelle fois au dualisme individus/institution, si ce n'est à une vision manichéenne (les bons et les méchants étant une partition commode pour le fonctionnement des groupes humains!).

Avoir exclu que le non sens et la crise puissent provenir des individus, ou d'un défaut d'institution et non pas seulement de ses excès, relève d'un déni des mécanismes pervers ou délirants pouvant agir dans les institutions, pouvant les saper de l'intérieur et les transformer en champ de bataille. Cette position de principe en recoupe une autre, très problématique, un refus assez obstiné d'envisager l'hypothèse d'une institution défaillante en tant qu'instance symbolique, une institution ne pouvant plus tenir et contenir ses ressortissants et réguler les significations et les affects. Ce déni procède ici aussi d'une coupure théorique entre l'institution et les sujets, toujours fantasmés ou représentés dans leur antagonisme, l'une - l'institution - étant la forme contraignante, l'autre - les sujets - la figure menacée par son ordre. Cette coupure justifierait alors qu'au nom des personnes, ces dernières étant plus ou moins confondues avec l'exercice démocratique, l'institué soit toujours subverti. 


\section{AssujetTissement Et/ou EMANCIPATION?}

Parallèlement aux théories "institutionnalistes", ${ }^{6}$ nous avons été très influencés durant les "jeunes" années de formation intellectuelle par l' approche foucaldienne ${ }^{7}$ et les technologies du pouvoir (techniques du corps, police, panoptique, subjectivation, enfermement, contrôle social, gestion des risques, bio-pouvoir etc.), qui ouvraient sur une perspective plus généalogique que strictement institutionnelle. Elles nous faisaient envisager les mécanismes d'assujettissement plus, certes, que l'émergence des individualités, en attendant un autre Foucault (2001), celui de ses derniers textes ouvrant explicitement cet espace théorique de la question du sujet. ${ }^{8}$

Le maillage théorique n'allait pas de soi - et c'est tout le génie de cette époque que de nous avoir autorisés à le penser - il s'agissait en effet de comprendre à la fois les logiques de pouvoir actualisées dans les institutions selon un mode conflictuel et dynamique (instituant contre institué) et les techniques de subjectivation exerçant une emprise sur les individus (assujettissement). Nous nous tirions de cet antagonisme par l'action politique, appelée tant pas l'analyse institutionnelle que par le foucaldisme.

Il faut également souligner que les théories féministes, très présentes dans les sciences humaines et sociales durant les années 1970-1980, furent déterminantes pour notre conscience des processus de “fabrique" des sujets. D'un côté les théories beauvoiriennes de l'assignation des femmes, soutenues par plusieurs anthropologues et sociologues semblaient irréfutables. D'un autre, de nombreuses contributions à la compréhension de l'histoire privée et intime des femmes, venaient à l'appui des travaux sur leur histoire dans l'espace public (Michèle Perrot en fut une des grandes initiatrices) et mettaient au jour l'existence d'un sujet femme ayant résisté aux diverses emprises socio-historiques.

Tenter de comprendre dans la période contemporaine les réagencements des rapports de sexe dans la société et dans la vie privée, de participer à la discussion sur la question de la différence sexuelle, alors que le principe de domination était fortement mis en question dans le champ politique, était particulièrement fécond pour les sociologues engagés par ailleurs dans ces transformations. Il s'agissait de lire un phénomène social en cours et dont nous participions: l'institutionnalisation de ce que portait et provoquait dans toute la société le mouvement d'émancipation des femmes. Ces transformations étaient en effet en train de s'inscrire au cœur de l'institué familial, de la vie privée, de l'intimité. Il m'intéressait particulièrement de porter attention à la dynamique très particulière qui se joue à ce moment précis de bascule d'un ordre ancien à un nouveau régime, et aux tensions qui s'y manifestent entre le "mouvement et l'institution" pour reprendre ici les catégories construites par Francesco Alberoni. Catégories qui, tout en étant fortement imprégnées de références à la sociologie tourainienne de l'action et des mouvements sociaux, ne sont pas sans évoquer celles de l'instituant, de l'institué et de l'institutionnalisation. 


\section{L'INSTITUTION DU SUJET COMME POSSIBLE DEPASSEMENT DU PARADIGME OPPRESSIF?}

L'approche de Pierre Legendre (1976) et sa théorisation de l'ordre dogmatique qui est, selon lui, aux fondements des institutions, pouvait servir de passerelle entre la critique des institutions et la psychanalyse. Sa lecture complétait en tous cas ce que les théories critiques de l'institution laissaient en panne. "Quelque chose, écrivait-il en 1976, fonctionne à l'insu des agents d'une institution quelle qu'elle soit, et commande l'homogénéité des conduites de ces mêmes agents, assimilés au point d'être, pour ainsi dire, interchangeables et stéréotypés" (LEGENDRE, 1976, p. 17). Nous sommes "possédés par les institutions, au point de les "avoir véritablement dans la peau" (LEGENDRE, 1976, p. 17), poursuit-il. La métaphore est forte. Il s'agissait de faire comprendre à une époque où tous les espoirs "révolutionnaires" étaient encore permis, les mécanismes faisant que les individus se rangent généralement dans cet ordre, qu'ils s'alignent sous la bannière de l'institution et n'en sont pas d'éternels révoltés ou insoumis. Mais à l'inverse, comme le constate aussi Legendre, la psychanalyse nous a fait clairement entrevoir que le sujet peut avoir "prise sur son aliénation" (LEGENDRE, 1976, p. 17). Au premier rang des mécanismes liant sujet et institutions figure cet "amour du censeur", 9 que Legendre (1974, p. 5) eût l'audace d'identifier, dans la filiation au droit canon, et alors que nous étions encore collectivement très marqués par la pensée soixante-huit et le désir d'émancipation tout azimut.

En proposant d'ancrer ainsi le sujet, Legendre s'éloigne de l'institution comme mode de contention des individus, de leurs affects et pulsions, et donc de la représentation d'une institution purement répressive, pour en faire un objet de la libido et du symbolique. La critique de la bureaucratie et des institutions méconnaissait la "servitude volontaire". Legendre nous rappelait comment l'institution se fait en quelque sorte aimer par la soumission des siens,${ }^{10}$ et produit des processus d'affiliation ayant entre autres fonctions la sublimation.

Dans sa quête de la fabrique du sujet aux confins de la psychanalyse et du droit, de la scène inconsciente et du texte juridique, l'institution dépasse le bon vouloir des sujets, ils n'en disposent pas, elle les fixe chacun à une place dans un ordre générationnel et sexué. La lecture legendrienne, même si elle ne constitue pas une référence définitive, permet de se saisir de ce concept d'institution des sujets. Pour qu'un sujet puisse émerger du magma sans significations que constituent a priori la vie pulsionnelle, les lois de l'espèce, les besoins et la profonde néoténie de l'enfant, il faut un certain nombre d'opérateurs, plus vastes et fondamentaux que les techniques éducatives mais aussi que le réseau des institutions socialisatrices. L'enfant est inscrit à une place dans la filiation, dans la généalogie, dans sa sexuation. Il prend place dans un réseau de significations sociales et familiales, sachant que le texte (les lois et codes juridiques, l'état civil) joue ici un rôle prépondérant, tout comme le langage dans la conception lacanienne: celui d'être porteurs de la Loi. Ces opérations sont constituées par les institutions (famille, institutions éducatives) qui à la fois le séparent, le protègent du fusionnel et le mettent en présence des autres et de ses pairs. Elles sont incarnées ou vectorisées 
par des fonctions symboliques servies et non pas définies par des individus - parents et éducateurs - qui sont là pour soutenir des limites face à l'enfant et transmettre les interdits et les prescriptions de la société.

\section{LE SUJET DE LA PSYCHANALYSE, SUJET DE L'INCONSCIENT}

La psychanalyse occupe une place de choix pour de nombreux chercheurs en sciences humaines depuis les années 1980. Elle s'inscrit dans la continuité et parachève de manière assez cohérente un itinéraire intellectuel commencé avec la question des institutions.

Le sujet au cœur duquel est inscrite la conflictualité (la conflictualité psychique telle qu'identifiée par Freud) et pour lequel on fait l'hypothèse de l'inconscient nous écarte d'une conception sociologique de l'acteur ou de l'agent social que la sociologie entend comprendre à travers ses pratiques concrètes et ses expériences objectives et subjectives du réel. Il ne peut y être question d'un sujet "entier", d'un sujet engagé de manière univoque dans ses actes et dans ses mots. Le sujet n'est pas maître à bord... il est soumis à des forces inconscientes. Une part de lui-même lui est obscure, échappe à sa raison et à son contrôle, mais se manifeste par des symptômes et des souffrances, des lapsus, des ratages, des actes manqués, des mots d'esprit.

Cette conception est directement liée au sujet parlant (le "parlêtre") et au hiatus que constitue forcément le langage. Il convient même de dire, depuis Lacan qui l'a ainsi explicité, que le sujet est celui de l'inconscient et du langage. Il est divisé "par l'effet de langage” (LACAN, 1973, p. 172), comme il l'écrit clairement dans Les quatre concepts fondamentaux de la psychanalyse.

L'approche lacanienne a consisté à faire comprendre cette division déjà constatée par Freud, mais aussi à insister sur le fait que le sujet n'est pas libre, qu'il est assujetti 'le sujet n'est sujet que d'être assujettissement au champ de l'Autre" (LACAN, 1973, p. 172).

Un tel sujet se révèlera en inadéquation avec les théories néo-libérales qui promeuvent un individu tout puissant, maître de son destin, élevé dans le culte de la performance et de l'individualisme. Les psychanalystes enregistrent, au contraire, les dégâts subjectifs de ce culte (LEBRUN, 1997, 2001).

Roland Chemama (1998) rappelle dans le Dictionnaire de la psychanalyse, que la psychanalyse met l'accent "non sur une subjectivité mais sur un assujettissement, entendons sur ce qui peut déterminer un sujet, le produire, le causer, sur son histoire et, plus précisément sur l'histoire d'un dire, celui qui était déjà là avant même sa naissance dans le discours de ses parents, celui qui depuis sa naissance n'a de cesse de l'accompagner et d'orienter sa vie dans un "tu es cela" sans échappatoire" (CHEMAMA, 1998b, p. 110) ${ }^{11}$.

Lacan avait tracé, dès 1938, les déterminations psychiques du sujet et leurs liens avec les "complexes familiaux": "La famille, écrivait-il, prévaut dans la première éducation, la répression des instincts, l' acquisition de la langue justement nommée maternelle. Par là, elle préside aux processus fondamentaux du 
développement psychique", "plus largement, elle transmet des structures de comportement et de représentation" (LACAN, 1938). La famille "établit ainsi entre les générations une continuité psychique dont la causalité est d'ordre mental". Cette continuité se manifeste "par la transmission à la descendance de dispositions psychiques qui confinent à l'inné" (LACAN, 1938, p. 13-14).

Ce n'est pas tant cet innéisme de la première période théorique de Lacan, que nous retiendrons comme significatif des déterminations du sujet, mais plutôt l'insécabilité qu'il suggère entre le sujet et l'Autre et qu'il s'emploiera à démontrer tout au long de ses séminaires et de ses Ecrits (LACAN, 1966). Le sujet se construit psychiquement sur la question du manque, construit sa relation d'objet, ce qui le fait sujet dans une dépendance au champ de l'Autre.

La psychanalyse nous pose finalement des questions similaires à celles soulevées lors de la réflexion critique sur l'institution. Elle amène en tous cas à penser, si ce n'est la dialectique du sujet, un paradoxe interne à son paradigme: la construction dynamique d'un sujet opère toujours en tension avec ses déterminismes psychiques. Ce paradoxe traverse toute la lecture que nous pouvons faire des textes de la psychanalyse, au travers de ses concepts qui opèrent un partage entre:

- d'une part, les dimensions dynamiques, celles qui montrent l'activité du sujet - je veux bien entendu parler ici d'activité psychique: le désir, les pulsions, la causalité psychique et la réalité psychique, la disposition psychique, l'identification, le choix de la psycho-névrose, le choix d'objet, l'éthique, la sublimation;

- et, d'autre part, les dimensions plus déterministes, celles qui balisent ce par quoi le sujet est assigné: les traumatismes, la division, les manques, l'inadéquation du langage, les signifiants, les complexes familiaux, l'emprise, l'aliénation, l'assujettissement, le refoulement, la pulsion de mort, le transgénérationnel, le symbolique.

Spontanément les freudiens sont plutôt perçus sous le premier angle, et les lacaniens sous le second. Mais comme Freud avait commencé à le faire en revisitant ses théories antérieures (la séduction, le trauma, les pulsions etc.), Lacan a brouillé les pistes, parce qu'il fait des concepts en apparence les plus déterministes (la castration symbolique, l'aliénation, la division) des éléments fondamentaux pour la "subversion du sujet et la dialectique du désir" (LACAN, 1966, p. 22), selon ses mots mêmes.

Pour chaque sujet existe une dynamique propre, faisant plus ou moins de place à l'expression de son désir, à sa capacité d'identification et de sublimation. Cette dynamique est enfreinte pour certains sujets, dès l'enfance, par une séparation impossible avec la mère, par un vacillement des limites, par un père absent à sa fonction, par la jouissance de la pulsion de mort, par des inhibitions et interdits de penser, par des peurs et des refus d'apprendre. Ce sont là autant d'entraves à ses divers apprentissages et à son inscription dans le lien social. Cela va, pour certains individus frappés de psychose jusqu'à évoquer l'idée d'une "mort du sujet" (CZERMAK, 2001). 
Confronté à tout ce qui le limite, à ce qui le contraint ou l'aliène à l'A Autre par le fait de sa dépendance et de ses manques, le sujet fait des petits choix quotidiens - ils sont "héroïques" écrit le psychanalyste Gérard Pommier (2001). ${ }^{12}$ La psychanalyse n'est en effet pas réductible à une théorie de l'assujettissement, elle nous amène à envisager aussi ce par quoi le sujet se construit, la part d' insoumission et de désir que cela suppose. Elle travaille précisément à faire émerger le sujet. "Wo Es War, soll Ich werden": là où c'était "je" doit advenir. Le sujet freudien est sans doute sujet, d'autant plus sujet, qu'il peut penser son état subjectif, ainsi que ses assujettissements. La psychanalyse invite à concevoir un sujet dégagé de ses entraves et de ses déterminations, paradoxalement parce qu'il se sait aliéné, qu'il connaît ce par quoi il est déterminé.

\section{LA CRISE DES SUBJECTIVITÉS AUJOURD’HUI}

Ce ne sont pas tant des questions strictement théoriques que l'apparition de nouvelles symptomatologies mi subjectives mi sociales, de nouvelles figures de la souffrance et de l'exclusion, mettant en difficulté les outils théoriques dont nous disposions qui m'ont récemment amenée à revisiter le concept d'institution et à envisager les conditions de son application et de son adéquation à la crise actuelle des subjectivités.

De nombreux observateurs relèvent aujourd'hui en effet dans la société contemporaine non pas les "exploits" de l'individu, libre de toute attache (religieuse, sexuelle, conjugale, familiale) tel qu' on a vu les revendiquer depuis 1968, mais une crise des sujets. L'individu de la post modernité souffre d'être "sans limites" (LEBRUN, 1997), et il connaît de ce fait des états de désarroi inédit. La dépression le menace.

Elisabeth Roudinesco (1999) ouvre son dernier ouvrage Pourquoi la psychanalyse?, par une partie intitulée "La société dépressive", suivie d'un premier chapitre sur la "défaite du sujet". Ce syndrome dépressif, Alain Ehrenberg (1998) le nomme la "fatigue d'être soi", alors que Marcel Gauchet (1998) qualifie de nouvelles "pathologies" de l'individu, les pathologies narcissiques si fréquentes selon lui actuellement. Les psychanalystes travaillant avec les jeunes évoquent un phénomène d'errance subjective et l'on constate de toute part la montée de la figure de l'enfant hyperactif présentant des troubles de l'attention. Sur son arrête sociale, le constat n'est pas plus réjouissant. Robert Castel (1976) identifie la montée des "individus par défaut" qu'il appelle aussi "individus négatifs", victimes de "désaffiliation". Quelques sociologues et juristes sont également préoccupés de ce qui se passe à l'articulation du sujet et du lien social, en particulier dans la sphère familiale et la filiation: la revue Esprit, par exemple, pointe régulièrement ces questions. ${ }^{13}$

Cette crise est rapprochée de la crise de la famille "moderne", et de ce que Marcel Gauchet appelle la désinstitutionnalisation de la famille, voire du désenchantement du monde. Bref, nous sommes loin du paradigme de l'oppression caractéristique des années 1970-1980. 
Il y a une sorte de contiguïté, dans les esprits et même dans le sens commun, entre les remaniements de la famille et la crise de l'autorité. Ce sont essentiellement les psychanalystes - d'obédience lacanienne - qui expriment la défaillance de l'autorité et ses effets subjectifs. La déclinaison de cette pensée s'appuie sur des concepts comme le "nom du Père" qui est au principe de la question symbolique et serait forclos dans la société contemporaine. Il est alors fortement question de brouillage ou de confusion des repères, voire des places symboliques.

Le discours social évoque lui aussi de manière récurrente la 'crise des repères', tandis que les professionnels de l'éducation et de la protection de l'enfance se font fréquemment l'écho d'une sorte de vulgate "psy" concernant la "défaillance paternelle", non sans que règne une certaine confusion des registres. On ne sait plus si ce qui est défaillant ce sont les pères en chair, un Père imaginé comme instance phallique, ou du Père comme séparateur et donc garant de la loi symbolique. Ces analyses sont également beaucoup reprises par les enseignants.

Tentons d'en proposer une autre lecture, notamment à la suite de Castoriadis et de comprendre les nouvelles dynamiques à l'œuvre.

L'ensemble des catégories que nous utilisons pour nommer, pour parler, pour désigner sont prises dans un réseau de significations sociales qui interviennent tant au plan imaginaire que symbolique. Et ce n'est sans doute pas abuser que de penser que la crise aujourd'hui vient frapper sur ce plan. Les significations ne sont pas ou plus partagées, ou sont mal partagées. Il n'est qu'à voir comment les observateurs que sont les intellectuels montrent des lectures divergentes du réel qui ne sont pas que des conflits de perspectives.

Dans son ouvrage des années 1970 - L'institution imaginaire de la société - Castoriadis (1975) montrait déjà combien les significations sociales imaginaires sont nécessaires à la société et aux individus car ce sont elles et/ou par elles qu'une société s'institue en instituant le sens, "un monde de significations" (CASTORIADIS, 1975, p. 482). Par là même, elles sont nécessaires à l'affiliation des individus dans un monde commun. Les mécanismes imaginaires jouant ici un rôle fédérateur pour le lien social et institutionnel.

Dans Les carrefours du labyrinthe, il va plus loin. Il souligne que via le langage et le symbolique, les institutions produisent de l'identitaire, de l'assemblage et de l'ensemble - ce qu'il appelle aussi une "auto-représentation de la société" faisant sortir du chaos ou de l'informe. Les individus "appartiennent" [sic] à une société parce qu'ils participent à ses significations imaginaires sociales, ils sont "façonnés, fabriqués par les institutions - c'est-à-dire par d'autres individus, euxmêmes porteurs de ces institutions et des significations corrélatives" (CASTORIDADIS, 1996, p. 20). Et c'est d'ailleurs à travers leur défaillance, qu'il repère dès les années 1980, que Castoriadis prophétise ce qu'il nomme une "montée de l'insignifiance". Autrement dit "la crise des sociétés occidentales contemporaines" n'est qu'une manière "de dire qu'il y a crise des significations imaginaires sociales, celles-ci ne fournissent plus aux individus les normes, valeurs, repères, motivations leur permettant à la fois de faire fonctionner la société, et de se maintenir eux-mêmes, tant bien que mal dans un "équilibre" vivable ("le 
'malheur banal' que Freud opposait à la 'misère névrotique"') (FREUD, 1981, p. 21). Par cette conception, Castoriadis, bien qu' "inventeur" de l'instituant, peut partager avec Mauss l'idée que le matériau des institutions est constitué de ce que l'anthropologue qualifiait d'

ensemble d'actes ou d'idées tout institué que les individus trouvent devant eux et qui s’impose plus ou moins à eux. [...] Nous entendons donc par ce mot aussi bien les usages et les modes, les préjugés et les superstitions que les constitutions politiques ou les organisations juridiques essentielles ; car tous ces phénomènes sont de même nature et ne diffèrent qu'en degrés (MAUSS ; FAUCONNET, 1971, p. 16-17).

S'il fait de la lutte pour l'autonomie des individus un des principaux moteurs de l'instituant, c'est-à-dire de la dynamique des institutions, Castoriadis n'est pas pour autant opposé à la fonction normative attendue de l'institution. Il propose d'ailleurs une théorie de l'aliénation assez radicalement différente de la conception marxiste et proche de la théorie lacanienne.

On ne peut réduire, l'aliénation à la répression des pulsions ou au conflit d'intérêt entre principe de plaisir et principe de réalité, ou aux antagonismes entre individu et société. Castoriadis rappelle à ce sujet une célèbre formule de Lacan "l'inconscient, c'est le discours de l'Autre" - et conclut que l'aliénation des individus relève de deux types de déterminants:

- les déterminants psychiques: l'aliénation est "pour une part décisive, le dépôt des visées, des désirs, des investissements, des exigences, des attentes, des significations dont l'individu a été l'objet dès sa conception et même avant, de la part de ceux qui l'ont engendré et élevé" (CASTORIADIS, 1975, p. 140).

- les déterminants socio-historiques qu'il nomme "l'inhérence du social". Cela revient à considérer l'hétéronomie (du social, des institutions, des significations imaginaires) comme nécessaire à l'autonomie des individus, celle-ci étant un rapport à construire par le sujet pour qu'il puisse soutenir son discours de sujet.

C'est sans aucun doute sous l'influence de la psychanalyse, et tout particulièrement de sa compagne Piera Aulagnier, que Castoriadis a pu penser la dimension symbolique et contenante de l'institution et pas seulement sa dimension disciplinaire.

Ce qui fait tenir ensemble les individus, ce qui fait contenant pour les subjectivités, ce qui vectorise le sujet, ce n'est évidemment pas ce qui contient au sens de la contention, réprimant les subjectivités et leurs expressions. Par contre cette instance contenante a à voir avec la répression des pulsions, avec ce que les psychanalystes (par exemple Michèle Montrelay, 1994) nomment "l'entame" du sujet dans sa toute puissance. La pulsion, les pulsions et leur contrôle ne peuvent relever, contrairement à l'idéologie dominante du consentement, ou de la libre activité des individus. Faut-il rappeler que le libéralisme économique le plus pur 
préconise la "liberté individuelle" comme principe régulateur du lien social (vous savez c'est la liberté du libre loup dans la bergerie...)?

L'autonomie ne peut se concevoir sans cette part d'aliénation du sujet. Et en pleine période "révolutionnaire" Castoriadis (1975, p. 155) écrivait qu'un sujet qui serait "délié de toute inhérence à l'histoire [...] qui aurait pris la tangente par rapport à la société - serait-ce en dominant exhaustivement son rapport à elle -, n'est pas un sujet autonome, c'est un sujet psychotique".

Si l'on reprend cette question du sujet et de l'institution dans l'actualité d'aujourd'hui, on perçoit bien que les institutions censées les soutenir, tout comme les figures et les fonctions éducatives, ont du mal à être suffisamment contenantes pour les individus, à jouer leur fonction de Tiers. De nombreux enfants et jeunes font symptôme de ce malaise. Lorsqu'ils viennent butter, par leur violence, leurs échecs ou leur dépression, sur des figures d'autorité introuvables et sur des dispositifs institutionnels en pleine mutation et dont manifestement la recomposition des significations leur échappe.

Dans un autre registre, plus directement économique et politique, les récents écrits de Castel (2001) sur la constitution de l'individualité dans la modernité et ses avatars actuels, nous éclairent sur ces processus que l'on pourrait qualifier de mi subjectifs, mi sociaux de désaffiliation. Pour exister comme individu, comme acteur ou comme sujet, Castel dit qu' "il faut avoir des supports", des "conditions objectives de possibilité", un "socle" qui vous donnent consistance dans la société, sachant que ce qui le constitue peut varier historiquement. Etre "propriétaire de soi", de sa personne, est connecté avec le fait d'être propriétaire (au sens de détenteur tel que conféré par la propriété privée) et/ou de jouir d'une "propriété sociale", c'est-à-dire être citoyen ressortissant de droits sociaux. Les droits sociaux jouent en effet pour les non nantis une fonction compensatoire par rapport à la pauvreté. C'est donc la dégradation de la propriété sociale aujourd'hui qui produit les "individus par défaut, ou individu que Castel qualifie aussi de "négatifs", caractérisés par leurs manques et défaillances en tous genres.

Dans les configurations très actuelles de la crise subjective, où l'économique tend sans cesse un peu plus ses injonctions à la performance et fait miroiter l'Individu comme figure de la réussite, continuer de soutenir à tout prix une position anti-institutionnelle peut rejoindre, sans le savoir, le libéralisme le plus sauvage et participer au déni des forces anomiques qui produisent précisément de la désaffiliation, en même temps que la promotion de la perversion au sein des institutions.

Partie des "origines" - la critique des institutions violentes et des forces d'oppression - arrivée à la crise contemporaine des subjectivités, nous avons tenté de rendre compte ici d'une trajectoire à la fois personnelle et collective à travers des théories et des concepts. Il ne s'agit point de tracer un lien de causalité, comme on l'entend souvent dans le discours social contemporain, entre les deux termes de cette trajectoire. 
Cette évolution, commune à beaucoup de chercheurs de la même génération, m'a particulièrement interrogée parce qu'elle nourrit aujourd'hui des représentations assez divergentes, si ce n'est des regrets, concernant le rapport entre le présent, le devenir et le passé, en particulier sur la question de l'autorité, de la transmission et du rapport entre les générations.

Deux positions extrêmes occupent le terrain des débats actuels en la matière.

Les uns, à l'éternelle jeunesse (!), continuent manifestement de penser le réel social en terme d'antagonismes toujours organisés sur le mode duel ou binaire mais finalement assez dénué de préoccupations politiques (institutions/ individus, société/sujets, ordre social/droits subjectifs, contraintes sociales/ libertés individuelles etc.), comme si l'histoire et leur propre maturation n'avaient point entamé ou poinçonné ces théories toujours présentées comme allant dans le sens du progrès. Les autres affectent de cultiver une sorte de nostalgie pour un ordre institué (que je nommerais volontiers "à la papa") relevant d'une construction quasi mythique selon laquelle seule la tradition - patriarcale - pourrait assurer l'ordre social et symbolique.

Comment alors penser bel et bien une troisième voie ? Une voie sortant des antagonismes au premier degré et où ne seraient pas confondus le plan du social et celui du symbolique, confusion entraînant que toute transformation de l'ordre social, par exemple des rapports de domination, soit perçue comme atteinte au symbolique!

L'hypothèse de l'instituant et très en lien avec elle, la théorie des analyseurs, ont été produites par l'observation des mouvements sociaux des années 1960 et par l'énergie qui se dégageait des collectifs permettant de penser le sens de l'histoire et des institutions autrement. Sans doute aujourd'hui avec la montée du libéralisme, avec la décomposition de bon nombre d'institutions sociales et politiques, sommesnous obligés de nous déplacer vers la question de l'institutionnalisation et vers les recompositions en train de se produire si nous ne voulons pas sombrer à notre tour dans la dépression et le no future ou devenir les gardiens d'un ordre social que nous avons espérer changer.

\section{Notas}

1 La sociologie française s'est assez longtemps arc-boutée sur ces grands dualismes opposant individus et société, acteurs et système, sujets et institutions, individuel et social. Selon Norbert Elias, on devrait considérer ces oppositions comme l' "ancien régime de la sociologie", sur lequel repose une vision assez dogmatique de la séparation disciplinaire entre sociologie et psychologie, notamment. Il nous invite à penser que le rapport entre "je et nous", n'est pas fixe et encore moins naturel. Le "je" (de l'individu) et le "nous" du collectif et leur rapport résultent de la société: il est construit socio-historiquement (ELIAS, 1987).

2 A l'occasion d'un travail universitaire présenté en vue de l'Habilitation, il me fallait reconstruire mon itinéraire de formation et de recherche. L'ambition n'était pas de produire pour s'en démarquer une nouvelle "pensée 68" à la manière de Ferry et Renaut, mais de retrouver quelques uns des outils avec lesquels nous avons, quoiqu'on en dise, appris à penser le monde et la société (GAVARINI, 2001). 
3 Comme nous avons pu le constater durant les années 1970-1980 à travers une expérience de la pratique socianalytique dans une quinzaine d'établissements principalement du secteur socioéducatif et médico-social.

4 La lecture de plusieurs psychosociologues comme Enriquez, René Kaes, Jean Dubost, ou de psychanalystes prenant en compte l'institutionnel et le groupe comme Guattari ou Gérard Mendel, faisait entrevoir - ils en avaient un peu l'exclusive à cette époque - la complexité du rapport sujets/groupes/institutions. Complexité dont Lapassade avait essayé de rendre compte en reprenant la dialectique sartrienne exposée dans Critique de la raison dialectique.

5 Réaliste, Castoriadis affirme qu'il y aura "toujours distance entre la société instituante et ce qui est, à chaque moment, institué, et cette distance n'est pas un négatif ou un déficit, elle est une des expressions de la créativité de l'histoire, ce qui l'empêche de se figer" (CASTORIADI, 1975, p. 156).

6 Ce sont revendiqués de cette appellation les praticiens de l'analyse institutionnelle et, de façon moindre, ceux de la psychothérapie institutionnelle et de la pédagogie institutionnelle.

7 Telle que pouvait nous l'enseigner Jacques Donzelot à propos de la famille (DONZELOT, 1977, 1984). Telle aussi que Foucault, lui-même, nous inclinait à la comprendre dans le cadre d'activités militantes au Groupe d'information sur les prisons (GIP).

8 Cf. en particulier L'estime de soi et le tout dernier recueil publié posthume L'herméneutique du sujet, cours au Collège de France, 1981-1982, Hautes Etudes, Gallimard, Seuil, 2001.

9 "Le Pouvoir touche au nœud du désir", écrit Pierre Legendre( 1974, p. 5) dans sa présentation de L'amour du censeur. Essai sur l'ordre dogmatique .

${ }^{10}$ Sur cette voie, comme le rappelle Legendre, Freud s'était risqué, s'appuyant lui-même sur un précédent célèbre, Gustave Le Bon et sa Psychologie des foules (1963), qui, avait mis en évidence le phénomène des meneurs des foules. Freud qui le commenta, non sans le critiquer, évoqua à son tour le "mirage", "l'illusion" d'un chef suprême et les "liens libidinaux" entre les individus d'une même foule (FREUD, 1981).

${ }^{11}$ Article "discours", in R. Chemana et B, Vandermerscer (sous la direction, Dictionnaire de la psychanalyse, Larousse, 1998, p. 110.

${ }^{12}$ Gérard Pommier (2001), évoque les "petits choix intimes qui ne sont pas toujours spectaculaires ou visibles, mais des petits choix intimes à faire presque tous les jours".

${ }^{13}$ Cf. par exemple Irène Théry, Catherine Labrusse, Denis Salas etc à propos du "Malaise dans la filiation" (1996).

\section{REFERÊNCIAS}

BASAGLIA, F. Les criminels de paix. Paris: PUF, 1976.

CASTEL, R. L'ordre psychiatrique: l'âge d'or de l'aliénisme. Paris: Minuit, 1976.

CASTEL, R.; HAROCHE, C. Propriété privée, propriété sociale, propriété de soi: entretiens sur la construction de l'individu moderne. Paris: Fayard, 2001.

CASTORIADIS, C. La crise des sociétés occidentales. In: CASTORIADIS, C. Les carrefours du labyrinthe IV. Paris: Seuil, 1996.

. L'institution imaginaire de la société. Paris: Seuil, 1975.

CHEMAMA, R.; VANDERMERSCH, B. (Dir.). Dictionnaire de la psychanalyse. Paris: Larousse, 1998a.

CHEMAMA, R.; VANDERMERSCH, B. (Dir.). Discours. In: . Dictionaire de la psychanalyse. Paris: Larousse, 1998b. p. 110. 
CZERMAK, M. Passions de l'objet: études psychanalytiques des psychoses. Paris: Association Freudienne Internationale, 2001.

DELEUZE, G. Pourparlers. Paris: Minuit, 1990.

DONZELOT, J. L’invention du social. Paris: Fayard, 1984.

. La police des familles. Paris: Minuit, 1977.

DURKHEIM, E. Les règles de la méthode sociologique. 11. ed. Paris: Presses Universitaires de France, 1949.

EHRENBERG, A. La fatigue d'être soi: dépression et société. Paris: Odile Jacob, 1998.

ELIAS, N. (1987/1991) La société des individus. Fayard, Agora, Pocket.

FOUCAULT, M. L'Herméneutique du sujet: Cours au Collège de France, 19811982. Paris: Gallimard: Seuil, 2001.

. Surveiller et punir. Paris: Gallimard, 1975.

FREUD, S. Psychologie des foules et analyse du moi. In: .. Essais de psychanalyse. Paris: Payot, 1981.

GAUCHET, M. Essai de psychologie contemporaine I: un nouvel âge de la personnalité. Le Débat, [S. 1.], n. 99, mars-avr. 1998.

GAVARINI, L. La formation des sujets: eléments pour une sociologie de l'enfance et des subjectivités. Paris: Université de Paris X, 2001.

GOFFMAN, E. Asiles: etude sur la condition sociale des malades mentaux. Paris: Minuit, 1968.

GUATTARI, F. Cartographies schizoanalytiques. Paris: Galilée, 1995.

LACAN, J. Les complexes familiaux. Paris: Navarin, 1938.

. Les écrits. Paris: Seuil, 1996.

. Séminaire, livre XI: les quatre concepts fondamentaux de la psychanalyse.

Paris: Seuil, 1973.

LE BON, G. La Psychologie des foules. Paris: PUF, 1963.

LEBRUN, J-P. Un monde sans limite: essai pour une clinique psychanalytique du social. Ramonville Saine-Agne: ERES, 1997.

2001.

(Dir.). Les désarrois nouveaux du sujet. Ramonville Sainte-Agne: ERES,

LEGENDRE, P. L'amour du censeur: essai sur l'ordre dogmatique. Paris: Seuil, 1974.

Les enfants du Texte: étude sur la fonction parentale des Etats: Leçons VI. Paris: Fayard, 1992. 
LEGENDRE, P. Jouir du pouvoir: traité de la bureaucratie patriote. Paris: Minuit, 1976.

LOURAU, R. L'analyse institutionnelle. Paris: Minuit, 1970. . Instituant contre institué. Paris: Anthropos, 1969.

MAUSS, M.; FAUCONNET, P. La sociologie, objet et méthode. In: MAUSS, M. Essais de sociologie. Paris: Minuit, 1971.

MENDEL, G. La sociopsychanalyse institutionnelle, pour qui? pour quoi? Paris: Petite Bibliothèque Payot, 1975.

MONTRELAY, M. L'entame paternelle. In: Che Vuoi? Revue de Psychanalyse, [S.1.], n. 2, p. 13-26, 1994..

OURY, F.; PAIN, J. Chronique de l'école caserne. Paris: Maspero, 1972.

POMMIER, G. La liberté du sujet. Confluences, [S. 1.], n. 51, Sept. 2001.

ROUDINESCO, E. Pourquoi la psychanalyse. Paris: Fayard, 1999.

SARTRE, J.-P. Critique de la raison dialectique. Paris: Gallimard, 1960.

THÉRY, I.; LABRUSSE, Catherine; SALAS, Denis. Malaise dans la filiation. Esprit [S. 1.], n. 227, p. 50-163, déc. 1996.

Recebido em: julho/2004 Aceito em: fevereiro/2005 